\title{
Application of Fuzzy Logic to Predictive Job Shop Scheduling in an Interconnected System
}

\author{
Onwuachu Uzochukwu C. \\ Department of Computer Sciences, \\ Imo state University, \\ Owerri, Nigeria.
}

\author{
P. Enyindah \\ Department of Computer Sciences, \\ University of Port Harcourt, \\ Choba, Nigeria.
}

\begin{abstract}
The features of high performance and reliability of systems have made them powerful computing tools. Such computing environment requires an efficient algorithm to determine when and on which system a given task should execute. This paper proposes a system that uses fuzzy logic in job allocation and job sequence or a dispatching rule in an interconnected system. The proposed system was implemented using MatLab 2008. It was designed to meet up with the timing, sequencing, routing and priority setting. The sequencing of jobs was approached using fuzzy controllers having rules with two antecedents which include the job processing time and the job Priority. From the result obtained, the system was able to achieve load balancing and minimize the job processing time.
\end{abstract}

\section{Keywords}

Fuzzy logic, job scheduling, job processing time, job Priority and interconnected system.

\section{INTRODUCTION}

Real-time systems are systems in which the time at which the result is produced is as important as the logical correctness of the result[11]. Scheduling is an important aspect in real-time systems to ensure soft and hard timing constraints. Scheduling tasks involves the allotment of resources and time to jobs, to satisfy certain performance needs. In a real-time application, real-time jobs are the basic executable entities that are scheduled. [10]. Scheduling a job set implies planning the order of execution of task requests so that the timing constraints are met. The schedule is said to be feasible if the timing constraints of all the tasks are met. All scheduling algorithms face the challenge of creating a feasible schedule. The main objective of task scheduling in real-time systems is achieving high resource utilization. Scheduling is the task that determines the job allocation and job sequence at each machine.

The Fuzzy scheduler considers the sequencing of job in the scheduling problem. The sequencing of jobs was approached using fuzzy controllers having rules with two antecedents which include the job processing time and the job Priority[1].The fuzzy inference system determine the priority of each job waiting in a system buffer, so that whenever the system are free the job with the highest priority among those waiting is chosen.

This paper proposed a fuzzy logic algorithm using fuzzy membership functions to model job scheduling problem. The solution sets will be pooled together and a final solution is obtained to minimize dissatisfaction amongst the decision makers.

\section{LITERATURE REVIEW}

Ziaul et al. (2012), flexible manufacturing systems (FMS) using a fuzzy-multicriteria based approach for job sequencing and routing. They applied Fuzzylogic based simulation and consider number variables with reasonable amount of accuracy.There proposed model prioritize the job and select the best alternative route with multicriteria scheduling through an approach based on a fuzzy logic. There are three criteria for both the jobsequencing and routing with 27 rules. With the help of the rules the sequence of the jobs are done and the best route is selected [1].

Marek and Roman (2015), worked on re-planning in predictive-reactive scheduling. They looked at the techniques related to predictive-reactive scheduling and suggest the future goal, which is to propose algorithms for dealing with unexpected events using the possibility of alternative processes [2].

Ramkumar et al, (2011), worked on multi criteria job shop schedule using fuzzy logic control for multiple machines multiple jobs. They use the amalgamation of fuzzy job shop scheduling approach to find profits, customer satisfaction and to solve the problem of vagueness and uncertainty using theory of fuzzy logic based in using a membership function tosolve a fuzzy mix product selection. They also present a theoretical model to demonstrate how fuzzy decision making can support the dynamic scheduling process, enabling the conflicting priorities of multi-objectives to bemanaged effectively in polynomial time [3].

Nagamalleswara et al, (2013), worked on modified heuristic time deviation technique for job sequencing and computation of minimum total elapsed time. From their work Job sequencing problem has become the major problem in the computer field. A finite set and each operation needs to be processed during an uninterrupted period of a given length on a given machine and our Purpose is to find a schedule, that is, anallocation of the operations to time intervals to machines that has minimal length.Thus their new modified heuristic technique called time deviation method is used to obtain the required job sequence and the minimum total elapsed time is also calculated for this sequence of jobs by the usual procedure [4].

Feng et al, (2008), worked on fuzzy logic based feedback scheduler for embedded control systems. A fuzzy logic based feedback scheduling approach where used to control multiple tasks sharing one embedded CPU. Execution times of these tasks and CPU workload are uncertain and imprecise. The sampling periods of control tasks are periodically adjusted with respect to uncertain resource availability. A simple period rescaling algorithm is employed, and the available 
CPU resource is dynamically allocated in an intelligent fashion. The proposed approach provides runtime flexibility to quality of control (QoC) management. Preliminary simulations highlight the benefits of the fuzzy logic based feedback scheduler [5]

Xiangzhen et al, (2010), worked onefficient dynamic task scheduling in virtualized data centers with fuzzy prediction. They proposed an efficient dynamic task scheduling scheme for virtualized data centers. Considering the availability and responsiveness performance, the general model of the task scheduling for virtual data centers is built and formulated as a two-objective optimization. A graceful fuzzy prediction method is given to model the uncertain workload and the vague availability of virtualized server nodes, by using the type-I and type-II fuzzy logic systems. A non-line dynamic task scheduling algorithm named SALAF is proposed and evaluated. Experimental results show that our algorithm can improve the total availability of the virtualized data center while providing good responsiveness performance [6]

Taravatsadat and Napsiah (2011), worked on application of artificial intelligent in production scheduling: a critical evaluation and comparison of key approaches, in their work Production scheduling is a part of operational research which relies on combinational optimization solved by discrete methods. Their review shows that there are only few research works which compare heuristic techniques on scheduling problem. There is a need for scholars to focus on evolutionary manufacturing systems, and hybrid models to face scheduling problem [7].

Rina and Harkut (2014), reviewed adaptive neuro fuzzy scheduler for real time task. Their work presents a review on scheduling algorithm of real time task. Then, discuss the limitations of EDF algorithm and features of neuro fuzzy system. They proposed neuro fuzzy scheduler using EDF to overcome the drawbacks of other algorithms for better scheduler performance of real time task [8].

\section{MATERIALS AND METHOD}

\subsection{The Basic Structure of a Fuzzy System.}

Figure 1 shows the basic structure of a fuzzy expert system. It has four key modules namely, fuzzification module, fuzzy inference engine, fuzzy rule-base and defuzzification module.

The fuzzification module is traditionally responsible for receiving crisp numeric measurements from the environment as input, process them and map them into fuzzy membership function values. The fuzzy engine is responsible for processing all calculated membership function values using fuzzy sets' calculations and communicates with fuzzy rule base to identify the most suitable fuzzy output. However, the defuzzification module is responsible for converting the fuzzy output into a numeric output suitable for the environment decision and control situation.

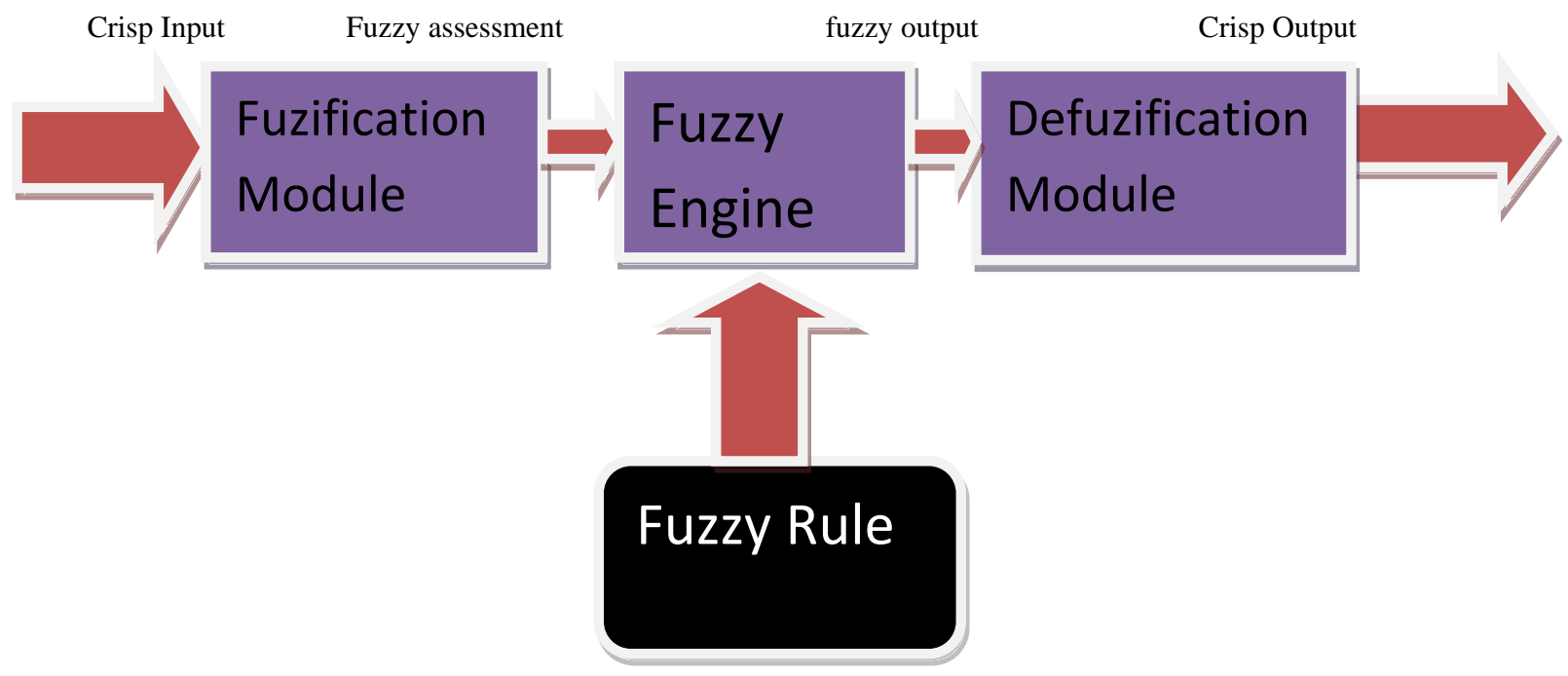

Figure 1: Basic Architecture of a Fuzzy Expert System

\subsection{The Proposed System}

The proposed system schedules the job in the queue based on the sequence of operations and the set of machines to be used to accomplish the operation. The fuzzy inference system calculates the membership function for the job priority and the job processing time which will be used in job allocation to different systems. The job priority is based on the importance of a job and based on the fuzzy values. The fuzzy sets the job priority JP which contains the five different fuzzy values. The customer priority fuzzy values are given as Bad, Low, Medium, High and Very Important Figure 2 shows the flow chart the proposed system. The job Processing Time is depending upon the processing time assigned to one particular job three fuzzy functions were assigned, for the fuzzy sets such as Short, Medium, and Long. 


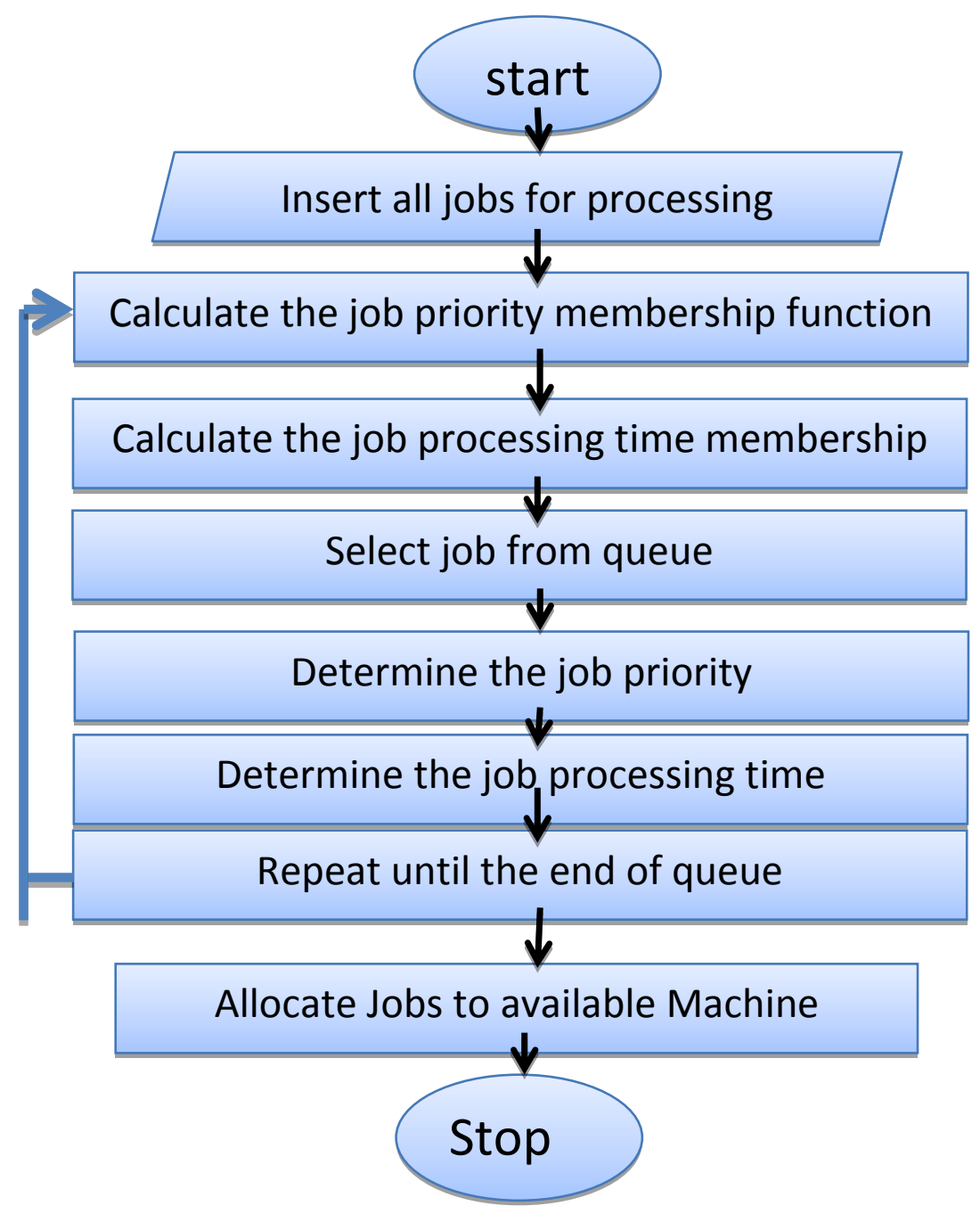

Figure 2: Flow chart of the proposed system

\section{EXPERIMENT AND RESULT}

The FIS Editor displays general information about a fuzzy inference system. It has a simple diagram at the top that shows the input variables on the left, and those of each output variable on the right. The sample membership functions shown in the boxes are just icons and do not depict the actual shapes of the membership functions. Figure 3 shows the fuzzy inference system editor for job scheduling.



Figure 3: The fuzzy inference system editor for job scheduling. 
The Membership Function Editor is the tool that lets you display and edits all of the membership functions associated with all of the input and output variables for the entire fuzzy inference system. The Membership Function Editor shares some features with the FIS Editor, as shown in the figure 4. In fact, all of the five basic GUI tools have similar menu options, status lines, and Help and Close buttons. From figure 3 we can see that the membership function for job allocation is displayed.

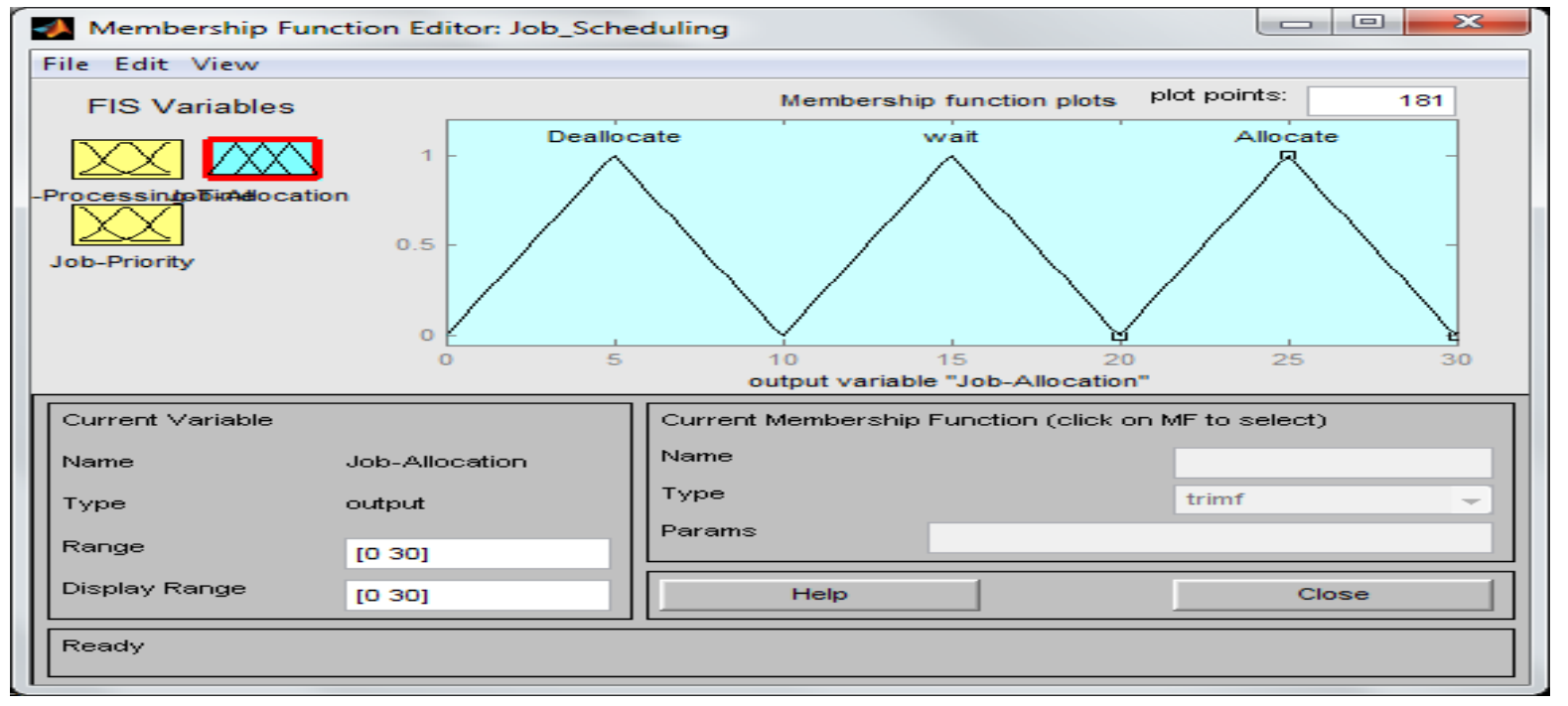

Figure 4: The fuzzy inference membership function editor for job scheduling.

Constructing rules using the graphical Rule Editor interface is fairly self-evident. Based on the descriptions of the input and output variables defined with the FIS Editor, the Rule Editor allows you to construct the rule statements automatically from the GUI. Figure 5 shows the fuzzy inference rule editor for job scheduling.



Figure 5: The fuzzy inference system rule editor for job scheduling. 
At this point, the fuzzy inference system has been completely defined, in that the variables, membership functions, and the rules necessary to calculate tips are in place. The Rule Viewer displays a roadmap of the whole fuzzy inference process. The Rule Viewer allows you to interpret the entire fuzzy inference process at once. It shows how the shape of certain membership functions influences the overall result. The Rule Viewer shows one calculation at a time and in great detail. In this sense, it presents a sort of micro view of the fuzzy inference system. Figure 6 shows the fuzzy inference rule viewer for job scheduling.

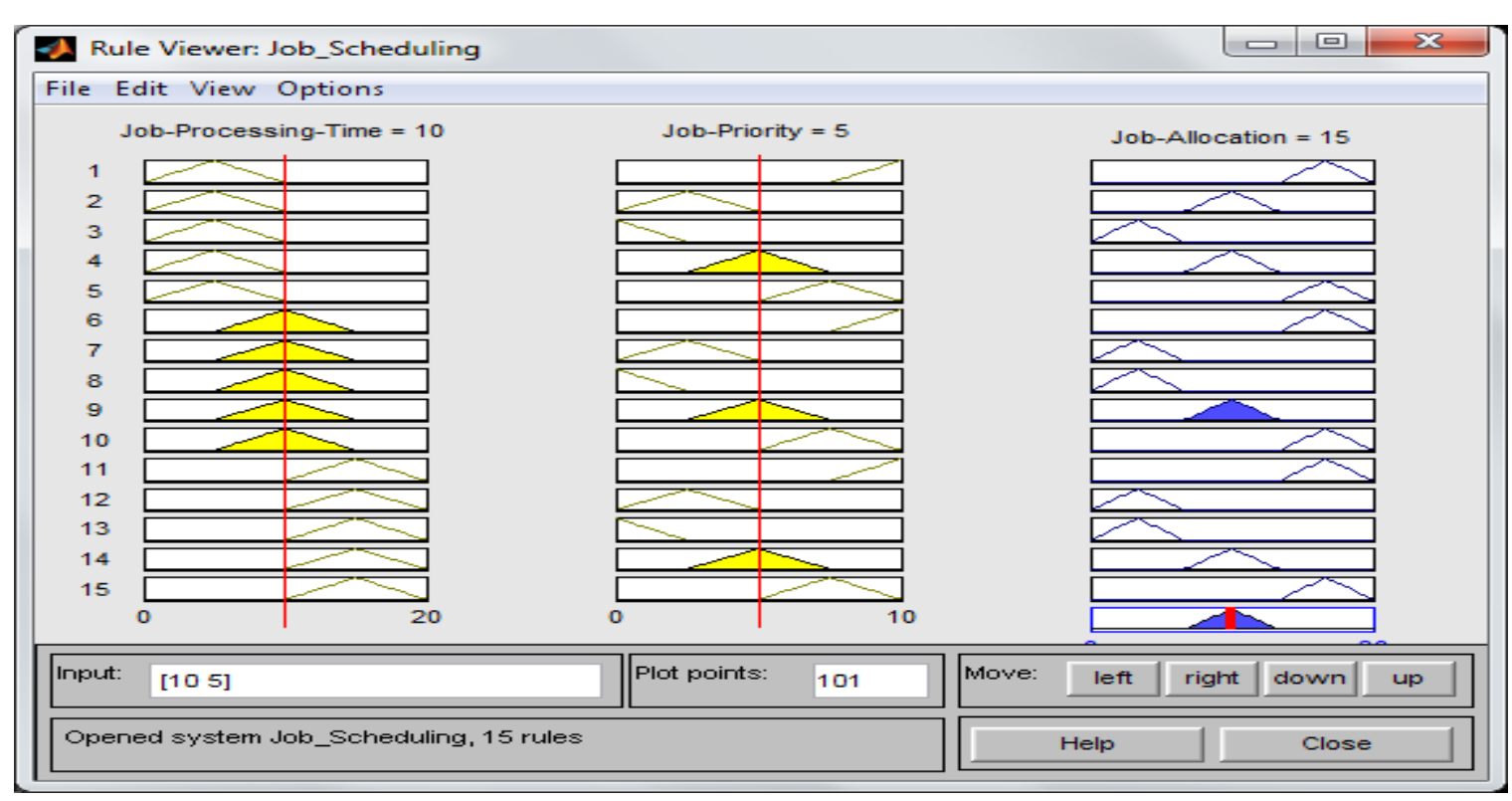

Figure 6: the fuzzy inference rule viewer for job scheduling.

The Surface Viewer has three-dimensional curve that represents the mapping from job priority and job processing time to job allocation. Because this curve represents a twoinput one-output case, you can see the entire mapping in one plot. Accordingly, the Surface Viewer is equipped with dropdown menus X (input):, $Y$ (input): and Z (output): that let you select any two inputs and any one output for plotting. Below these menus are two input fields $\mathrm{X}$ grids: and $\mathrm{Y}$ grids: that let you specify how many $\mathrm{x}$-axis and $\mathrm{y}$-axis grid lines you want to include. Figure 7 shows the fuzzy inference surface viewer for job scheduling.

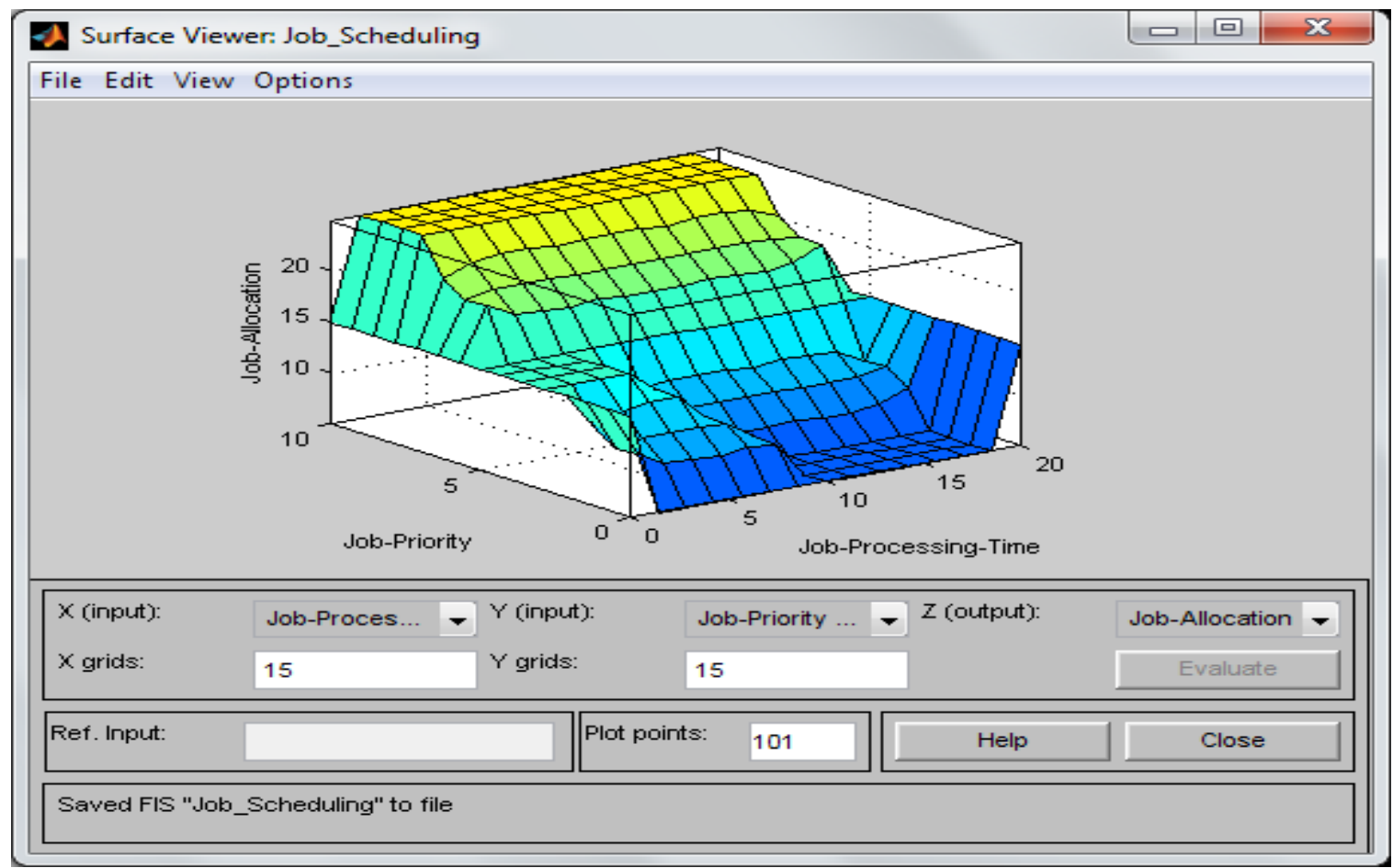

Figure 7: The fuzzy inference surface viewer for job scheduling. 


\section{RESULT DISCUSSION}

Figure 3 shows the fuzzy inference system editor for job scheduling. The FIS Editor displays general information about a fuzzy inference system. It used to edit the input and the output of the fuzzy inference system. The Membership Function Editor is the tool that lets you display and edits all of the membership functions associated with all of the input and output variables for the entire fuzzy inference system. From figure 4 we can see how the membership function for job allocation is displayed.

Figure 5 shows the fuzzy inference rule editor for job scheduling. Constructing rules using the graphical Rule Editor interface is fairly self-evident. Based on the descriptions of the input and output variables defined with the FIS Editor, the Rule Editor allows you to construct the rule statements automatically from the GUI. The Rule Viewer displays a roadmap of the whole fuzzy inference process. The Rule Viewer allows you to interpret the entire fuzzy inference process at once. It shows how the shape of certain membership functions influences the overall result as it is shown in figure 6.The Surface Viewer has three-dimensional curve that represents the mapping from job priority and job processing time to job allocation. Because this curve represents a two-input one-output case, you can see the entire mapping in one plot. Figure 7 shows the fuzzy inference surface viewer for job scheduling

\section{CONCLUSION}

The paper has proven that fuzzy logic system can be used to achieve efficacy and load balancing in job allocation and job sequence within an interconnected systems. The Scheduling algorithms are considered one of the key components of a real-time system.A fuzzy scheduling algorithm builds into the real-time system flexibility and adaptation to the uncertainty inherent in real-time environments and offers a means to improve several important characteristics of real-time systems.

\section{REFERENCES}

[1] Ziaul Hassan, Nabila Chowdhury, Abdullah-AlMamunMasud (2012), A Fuzzy-Multicritaria Based Approach for Job Sequencing and Routing In Flexible Manufacturing System (Fms), Global Journal of Researches in Engineering Mechanical and Mechanics Engineering, Volume 12 Issue 5 Version 1.0, Online ISSN: 2249-4596 Print ISSN:0975-5861

[2] Marek Vlk and Roman Barták (2015), Replanning in Predictive-reactive Scheduling, Association for the Advancement of ArtificialIntelligence (www.aaai.org).
[3] R. Ramkumar, Dr. A. Tamilarasi and Dr. T. Devi (2011), Multi Criteria Job Shop Schedule Using Fuzzy Logic Control for Multiple Machines Multiple Jobs, International Journal of Computer Theory and Engineering, Vol. 3, No. 2, April 2011 ISSN: 1793-8201

[4] Nagamalleswara Rao, Dr. O. Naga Raju and Prof. I. Ramesh Babu (2013), modified heuristic time deviation technique for job sequencing and computation of minimum total elapsed time,International Journal of Computer Science \& Information Technology (IJCSIT) Vol 5, No 3,

[5] Feng Xia, Xingfa Shen, Liping Liu, Zhi Wang, and Youxian Sun (2008), Fuzzy Logic Based Feedback Scheduler for Embedded Control Systems, National Laboratory of Industrial Control Technology

[6] Xiangzhen Kong, Chuang Lin , Yixin Jiang, Wei Yan, Xiaowen Chu (2010), Efficient dynamic task scheduling in virtualized data centers with fuzzy prediction, Journal of Network and Computer Applications, www.elsevier.com/locate/jnca.

[7] TaravatsadatNehzati and Napsiah Ismail (2011) Application of Artificial Intelligent in Production Scheduling: a critical evaluation and comparison of key approaches, Proceedings of the 2011 International Conference on Industrial Engineering and Operations ManagementKuala Lumpur, Malaysia.

[8] Rina V. Bhuyar and Harkut D. G. (2014), Adaptive Neuro Fuzzy Scheduler for Real Time Task: A Review, International Journal of Advanced Research in Computer Science and Software Engineering, Volume 4, Issue 2, ISSN: $2277128 \mathrm{X}$

[9] Paolo Dadone (1997), Fuzzy Control of Flexible Manufacturing Systems, Thesis submitted to the Faculty of the Virginia Polytechnic Institute and State University in partial fulfillment of the requirements for the degree of Master of Science in Electrical Engineering.

[10] Saad E. M., Keshk H. A., Saleh M. A., and Hamam.A.A,(2009), Scheduling Real-Time Tasks In Multiprocessor Systems Using Genetic Algorithms, Journal of Engineering Sciences, Assiut University, Vol. 37, No. 3, pp. 691-698

[11] Aparna Vishwanath1, Ramesh Vulavala, Sapna U. Prabhu (2014), Task Scheduling in Homogeneous Multiprocessor Systems Using Evolutionary Techniques, International Journal of Emerging Technology and Advanced Engineering, ISSN 2250-2459, Volume 4, Issue 2 , 